\title{
MANEJO DO PACIENTE EM PRIMEIRO EPISÓDIO DE SURTO PSICÓTICO
}

\section{ARTIGO DE REVISÃo}

FERNANDES JÚNIOR, Pedro Bonifácio ${ }^{1}$, LIMA, Adriely Portela Souza², OURIQUES, Caio Flávio Nascimento Mendes ${ }^{3}$, ALMEIDA, Caroline Duarte de $^{4}$, TORRES, Cibelle da Silva ${ }^{5}$, MINARI, Fernando de Lima $^{6}$, MAIA, Juliana Marino ${ }^{7}$, SANTOS, Larissa Rodrigues Cardoso ${ }^{8}$, LIZ, Lorena Ponzetto Rosilho de ${ }^{9}$, BRITO, Lucas Pinheiro ${ }^{10}$, PEREIRA, Priscilla Nery Tavares ${ }^{11}$, OLIVEIRA, Igor de Sousa ${ }^{12}$, RIBEIRO, Yana Sarah Fernandes Souza ${ }^{13}$, VASCONCELOS, Gilberto Loiola de ${ }^{14}$

FERNANDES JÚNIOR, Pedro Bonifácio. Et al. Manejo do paciente em primeiro episódio de surto psicótico. Revista Científica Multidisciplinar Núcleo do Conhecimento. Ano 06, Ed. 07, Vol. 12, pp. 22-42. Julho de 2021. ISSN: 2448-0959, Link de acesso: https://www.nucleodoconhecimento.com.br/saude/surto-psicotico, DOI: 10.32749/nucleodoconhecimento.com.br/saude/surto-psicotico

\section{RESUMO}

Objetivo: Analisar e comparar abordagens terapêuticas de manejo precoce em pacientes em primeiro episódio de surto psicótico. Métodos: Trata-se de revisão

\footnotetext{
${ }^{1}$ Discente do curso de Medicina da Universidade Federal de Juiz de Fora.

2 Discente do curso de Medicina da Universidade Nilton Lins.

${ }^{3}$ Discente do curso de Medicina do Centro Universitário INTA - UNINTA.

${ }^{4}$ Discente do curso de Medicina da Universidade Federal do Pará.

${ }^{5}$ Discente do curso de Medicina do Centro Universitário INTA - UNINTA.

${ }^{6}$ Discente do curso de Medicina da Universidade Nove de Julho - UNINOVE, Campus Guarulhos.

${ }^{7}$ Discente do curso de Medicina da Universidade Federal de Juiz de Fora, Campus Governador Valadares.

${ }^{8}$ Discente do curso de Medicina da Universidade de Itaúna.

${ }^{9}$ Discente do curso de Medicina do Centro Universitário São Lucas.

${ }^{10}$ Discente do curso de Medicina do Centro Universitário INTA - UNINTA.

${ }^{11}$ Discente do curso de Medicina do Centro Universitário INTA - UNINTA.

12 Discente do curso de Medicina da Universidade Federal de Campina Grande, Centro de Formação dos Professores, Cajazeiras - Paraíba.

${ }^{13}$ Discente do curso de Medicina do Centro Universitário INTA - UNINTA.

${ }^{14}$ Orientador. Médico residente em Clínica Médica, formado pela Universidade Federal do Ceará.
}

RC: 92275

Disponível em: https://www.nucleodoconhecimento.com.br/saude/surto-psicotico 
bibliográfica integrativa de 11 artigos disponibilizados na base de dados Biblioteca Virtual de Saúde (BVS) a partir de fórmula de busca constituída por descritores disponíveis no DeCS (Descritores em Ciências da Saúde). Resultados: Dentre os estudos selecionados, $18,1 \%(n=2)$ evidenciaram que o tratamento intensivo especializado, por 24 meses, é conduta imprescindível na abordagem do primeiro surto psicótico. Outros $18,1 \% \quad(n=2)$ evidenciaram a relevância da terapia de recuperação social, uma adaptação da terapia cognitivo-comportamental que atesta melhor prognóstico e melhores resultados no desfecho do primeiro episódio psicótico. Ademais, $18,1 \%(n=2)$ apontaram desfechos favoráveis em relação às terapias de remediação cognitiva em perfis de pacientes específicos. Conclusão: $O$ estudo evidenciado a importância da abordagem precoce e multidisciplinar ao paciente em primeiro episódio de surto psicótico, bem como o acompanhamento intensivo em momentos posteriores ao desenvolvimento do primeiro episódio do surto psicótico.

Palavras-chave: Transtornos Psicóticos, Procedimentos Terapêuticos, Tratamento Multimodal.

\section{INTRODUÇÃO}

O primeiro episódio de surto psicótico é uma desordem mental de início repentino e uma emergência médica, que pode estar vinculado ou não com doenças psíquicas, como a esquizofrenia e o uso de substâncias químicas. De acordo com a Organização Pan-Americana da Saúde (ANO), a genética, o estresse, a nutrição e as infecções perinatais são alguns fatores que podem favorecer o aparecimento de transtornos mentais.

As repercussões clínicas do Primeiro Episódio Psicótico (PEP) são delírios, alucinações, agitação psicomotora, pensamento e comportamento desordenado. Além dessa sintomatologia, é necessária investigar as correlações existentes, uma vez que pode estar associado a fatores agravantes, a exemplo de sinais de

RC: 92275

Disponível em: https://www.nucleodoconhecimento.com.br/saude/surto-psicotico 
depressão, transtorno mental, etilismo e outras drogas. Cita-se que o episódio deve ser inédito e não deve ter havido remissão do quadro desde o início dos sintomas.

Cabe salientar também que antes de apresentar a fase aguda, a pessoa apresenta uma fase prodrômica, a qual só pode ter o diagnóstico após o surto psicótico. De fato, a identificação dessa fase é difícil, visto que há indefinição do quadro sintomático.

Em virtude da dificuldade de se firmar um diagnóstico inicial no PEP e pelo fato de que esse diagnóstico quando erroneamente estabelecido pode causar graves implicações terapêuticas e prognósticas para os pacientes, deve-se ensejar e compreender todas as particularidades do manejo em prol de maximizar a possibilidade diagnóstica. Diante disso, surge a seguinte questão: "Qual manejo mais indicado para o paciente no Primeiro Episódio Psicótico?".

De fato, atualmente, o diagnóstico do PEP é realizado por meio de entrevista clínica estruturada, baseada em critérios diagnósticos do DSMIV-TR e do CID-10. Nos casos de PEP, o mais importante e específico biomarcador para auxílio ao diagnóstico nosológico é a dosagem sérica de substâncias psicoativas em casos em que a clínica e a história sugerem sua confirmação para o diagnóstico mais preciso. Outrossim, pacientes em PEP evidenciam aumento de dano oxidativo medidos por meio de menor concentração de glutationa e aumento da peroxidação lipídica.

Evidências crescentes apontam que alterações de estruturas cerebrais ocorrem em pacientes em PEP e que algumas dessas alterações evoluem sobretudo nos anos iniciais após o início do quadro psicótico, sendo necessário a atenção prioritária no diagnóstico e no tratamento desses pacientes Isso evidencia-se em numerosos serviços de intervenção precoce direcionados a jovens com psicose foram estabelecidos em todo o mundo nas últimas duas décadas, com base na premissa de que reduzir o atraso do tratamento e fornecer intervenções intensivas específicas da fase nos primeiros anos de psicose pode melhorar o desfecho da doença em longo prazo.

RC: 92275

Disponível em: https://www.nucleodoconhecimento.com.br/saude/surto-psicotico 
O programa de intervenção Serviço de Avaliação Antecipada para Jovens com Psicose (EASY) foi lançado em 2001 e inclui programas de sensibilização da comunidade, um sistema de referência aberto e uma intervenção especializada de 2 anos para jovens com primeiro episódio de psicose, seguido de 1 ano cuidados reduzidos com acompanhamento médico preservado, mas sem prestação de serviço de gerenciamento de caso A avaliação do programa EASY usando métodos de controle histórico mostrou que os pacientes que receberam intervenção precoce tiveram melhor funcionamento, gravidade dos sintomas mais leves, menos suicídios e internações hospitalares (PETERSEN et al., 2008).

Diante do exposto, fica clara a importância dos estudos com respeito ao manejo de pacientes em primeiro episódio de surto psicótico. Essa correlação está presente devido aos maiores impactos físicos e psicológicos em que podem estar clinicamente ligados ao manejo correto da equipe multidisciplinar e à abordagem ao paciente no primeiro momento, aumentando substancialmente benefícios na melhor qualidade de vida do paciente

Desse modo, esse estudo tem como objetivo analisar os tipos de tratamentos e dos serviços na primeira abordagem ao paciente com surto psicótico.

\section{METODOLOGIA}

Trata-se de uma revisão bibliográfica integrativa, retrospectiva, do tipo quantiqualitativa e destinada a analisar e descrever estudos nacionais e internacionais. Enseja-se descrever as perspectivas do manejo clínico de paciente em primeiro episódio de surto psicótico.

Foram utilizadas como bases de dados, para a busca bibliográfica, a Biblioteca Virtual de Saúde (BVS) e seus sites aliados. Primeiramente, foi realizada uma confirmação dos descritores apropriados na busca de artigos relevantes para o trabalho por meio do vocabulário disponível no DeCS (Descritores em Ciências da Saúde), sendo esses: "transtornos psicóticos", "diagnóstico" e "terapêutica". Para o

RC: 92275

Disponível em: https://www.nucleodoconhecimento.com.br/saude/surto-psicotico 
levantamento dos artigos, tais descritores possibilitaram, associado ao inglês e ao espanhol, a configuração da seguinte fórmula de busca: "(transtornos psicóticos) AND (diagnóstico) AND (terapêutica)".

Dessa forma, baseado no modelo de busca acima, foi encontrado um total de 31 artigos nas referentes bases de dados consultadas. Com isso, elegem-se os critérios de inclusão, sendo esses: artigos publicados em português, inglês e espanhol; artigos disponíveis na íntegra que descrevessem tópicos relacionados a revisões abrangentes e artigos publicados e indexados nos referidos bancos de dados há pelo menos 10 anos, no intuito de enfocar as referências mais inéditas e relevantes. Além disso, foram excluídos os artigos indisponíveis na íntegra, repetidos e que não atendiam os objetivos da pesquisa.

Por conseguinte, após a leitura do título e do resumo dos artigos encontrados por parte dos pesquisadores, ISO e LPB, bem como em associação com os critérios previamente citados, apenas 11 dos 31 artigos foram incluídos.

Deste modo, é importante destacar que para esse estudo não foi necessário a submissão ao Comitê de Ética em Pesquisa (CEP), visto que a pesquisa não tem aplicação prática e, consequentemente, não houve, de forma direta ou indireta, manipulação de seres humanos.

Nessa perspectiva, tanto a análise quanto a síntese dos dados extraídos dos artigos estão retratados de forma descritiva, visto que permitiram a configuração de uma tabela contendo as principais informações dos artigos que foram revisados na pesquisa, sendo estas: título, autores, ano de publicação, e os principais resultados, com intuito de reunir o conhecimento gerado sobre o tema explorado na revisão.

RC: 92275

Disponível em: https://www.nucleodoconhecimento.com.br/saude/surto-psicotico 


\section{RESULTADOS}

Tabela 1: Principais Resultados dos Artigos Selecionados para Pesquisa

\begin{tabular}{|c|c|c|c|}
\hline & Título & $\begin{array}{l}\text { Autores, ano } \\
\text { de } \\
\text { publicação e } \\
\text { país }\end{array}$ & Principais Resultados \\
\hline ARTIGO & $\begin{array}{l}\text { Optimal duration of an early } \\
\text { intervention programme for } \\
\text { first-episode psychosis: } \\
\text { randomised controlled trial. }\end{array}$ & $\begin{array}{l}\text { Chang et al., } \\
2015 \text {, China. }\end{array}$ & $\begin{array}{l}\text { Pacientes } \\
\text { realizaram tratamento } \\
\text { intensivo de surto } \\
\text { psicótico em período } \\
\text { maior que dois anos } \\
\text { atingiram melhorias } \\
\text { funcionais } \\
\text { sintomáticas. }\end{array}$ \\
\hline $\begin{array}{l}\text { ARTIGO } \\
\text { II }\end{array}$ & $\begin{array}{l}\text { Trans-Sector Integrated } \\
\text { Treatment in Psychosis and } \\
\text { Addiction. }\end{array}$ & $\begin{array}{l}\text { Gouzoulis- } \\
\text { Mayfrank et } \\
\text { al., 2015, } \\
\text { Alemanha. }\end{array}$ & $\begin{array}{l}\text { Observou-se que a } \\
\text { utilização de tratamento } \\
\text { integrado, motivacional } \\
\text { e com elementos } \\
\text { terapêuticos } \\
\text { psicoeducativos } \\
\text { comportamentais são } \\
\text { úteis na intervenção } \\
\text { hospitalar padrão em } \\
\text { pacientes com psicose. }\end{array}$ \\
\hline $\begin{array}{l}\text { ARTIGO } \\
\text { III }\end{array}$ & $\begin{array}{l}\text { Psychological interventions } \\
\text { for psychosis in adolescents. }\end{array}$ & $\begin{array}{l}\text { Datta et al., } \\
\text { 2020, Reino } \\
\text { Unido. }\end{array}$ & $\begin{array}{l}\text { Demonstrou-se que na } \\
\text { psicose em } \\
\text { adolescentes a terapia } \\
\text { de remediação cognitiva } \\
\text { é preferível para a }\end{array}$ \\
\hline
\end{tabular}

RC: 92275

Disponível em: https://www.nucleodoconhecimento.com.br/saude/surto-psicotico 


\begin{tabular}{|c|c|c|c|}
\hline & & & $\begin{array}{l}\text { manutenção do } \\
\text { funcionamento } \\
\text { cognitivo. Atestou-se, } \\
\text { ainda, que a terapia } \\
\text { psicossocial de grupo é } \\
\text { favorável ao estado } \\
\text { global do paciente. }\end{array}$ \\
\hline $\begin{array}{l}\text { ARTIGO } \\
\text { IV }\end{array}$ & $\begin{array}{l}\text { An intensive multimodal group } \\
\text { programme for patients with } \\
\text { psychotic disorders at risk of } \\
\text { rehospitalization: a controlled } \\
\text { intervention study. }\end{array}$ & $\begin{array}{l}\text { De Jong et } \\
\text { al., 2019, } \\
\text { Holanda. }\end{array}$ & $\begin{array}{lr}\text { Identificou-se, no grupo } \\
\text { de } & \text { pacientes } \\
\text { submetidos } & \text { ao } \\
\text { programa } & \text { intensivo } \\
\text { multimodal, } & \text { que } \\
\text { pacientes } & \text { mais } \\
\text { propensos à internação } & \\
\text { hospitalar passaram } \\
56,1 \% \text { menos tempo em } \\
\text { internação psiquiátrica } \\
\text { do que os indivíduos do } \\
\text { grupo-controle. }\end{array}$ \\
\hline $\begin{array}{l}\text { ARTIGO } \\
\mathrm{V}\end{array}$ & $\begin{array}{l}\text { COllaborative Shared care to } \\
\text { IMprove Psychosis Outcome } \\
\text { (COSIMPO): study protocol } \\
\text { for a randomized controlled } \\
\text { trial }\end{array}$ & $\begin{array}{l}\text { Gureje et al., } \\
2017 \text {, Nigéria. }\end{array}$ & $\begin{array}{l}\text { Constatou-se que } \\
\text { políticas destinadas a } \\
\text { envolver formalmente } \\
\text { curandeiros tradicionais } \\
\text { e religiosos dentro do } \\
\text { sistema de saúde } \\
\text { devem ser discutidas, } \\
\text { pois podem propiciar } \\
\text { melhores desfechos } \\
\text { clínicos ao paciente. }\end{array}$ \\
\hline ARTIGO & The RAISE Early Treatment & Kane et al., & Concluiu-se \\
\hline
\end{tabular}

RC: 92275

Disponível em: https://www.nucleodoconhecimento.com.br/saude/surto-psicotico 
V

\begin{tabular}{ll|l|l} 
Program for First-Episode 2015, & programa de tratamento
\end{tabular}
Psychosis: Background, Estados precoce de primeiro Rationale, and Study Design. Unidos da episódio de surto América. psicótico (RAISE) pode ser realizado em ambientes clínicos e impactar diretamente no processo clínico da Esquizofrenia em fase inicial.

ARTIGO Multimodal prevention of first

Lowen et al., Demonstrou-se a

VII psychotic episode through $\mathrm{N}$ acetyl-L-cysteine and 2019, Suíça.

importância da Integrated Preventive implantação de Psychological Intervention in protocolos que individuals clinically at high objetivem identificar as risk for psychosis. Protocol of intervenções mais a randomised, placeboeficazes e toleráveis relacionadas à transição controlled, parallel- group do estado clínico para a trial. psicose.

\begin{tabular}{l|l|l|lr} 
ARTIGO & Cognitive Remediation Can & Ventura et al., & Observou-se & que \\
VIII & Improve Negative Symptoms & 2017, & pacientes & com \\
& and Social Functioning in & Estados & & esquizofrenia em fase \\
& First-Episode Schizophrenia: & Unidos da & inicial & possuem \\
A Randomized Controlled & América. & melhoras nos sintomas \\
& Trial & & negativos quando em \\
& & & uso da Remediação \\
& & & Cognitiva (RC).
\end{tabular}

\begin{tabular}{l|lr|lrl} 
ARTIGO & Predictors of treatment & Wessels et & Verificou-se & que \\
IX & response to psychological & al., 2017, & intervenções &
\end{tabular}

RC: 92275

Disponível em: https://www.nucleodoconhecimento.com.br/saude/surto-psicotico 


\begin{tabular}{|c|c|c|c|}
\hline & $\begin{array}{l}\text { interventions in people at } \\
\text { clinical high risk of first- } \\
\text { episode psychosis. }\end{array}$ & Alemanha. & $\begin{array}{l}\text { psicológicas podem ser } \\
\text { benéficas em pacientes } \\
\text { mais jovens com alto } \\
\text { risco clínico de } \\
\text { desenvolvimento de } \\
\text { surto psicótico. }\end{array}$ \\
\hline $\begin{array}{l}\text { ARTIGO } \\
x\end{array}$ & $\begin{array}{l}\text { Measuring adherence in } \\
\text { social recovery therapy with } \\
\text { people with first episode } \\
\text { psychosis. }\end{array}$ & $\begin{array}{l}\text { Lowen et al., } \\
\text { 2020, Reino } \\
\text { Unido. }\end{array}$ & $\begin{array}{l}\text { Atestou-se significativa } \\
\text { relevância da } \\
\text { abordagem } \\
\text { comportamental na } \\
\text { Terapia de } \\
\text { Recuperação Social de } \\
\text { indivíduos em primeiro } \\
\text { episódio de surto } \\
\text { psicótico. }\end{array}$ \\
\hline $\begin{array}{l}\text { ARTIGO } \\
\mathbf{X I}\end{array}$ & $\begin{array}{l}\text { Staged treatment and } \\
\text { acceptability guidelines in } \\
\text { early psychosis study } \\
\text { (STAGES): A randomized } \\
\text { placebo controlled trial of } \\
\text { intensive psychosocial } \\
\text { treatment plus or minus } \\
\text { antipsychotic medication for } \\
\text { first-episode psychosis with } \\
\text { low-risk of self-harm or } \\
\text { aggression. Study protocol } \\
\text { and baseline characteristics } \\
\text { of participants }\end{array}$ & $\begin{array}{l}\text { O'Donoghue } \\
\text { et al., 2018, } \\
\text { Austrália. }\end{array}$ & $\begin{array}{l}\text { Concluiu-se que será } \\
\text { determinado se } \\
\text { medicamentos } \\
\text { antipsicóticos são } \\
\text { indicados para jovens } \\
\text { com primeiro episódio } \\
\text { de psicose (FEP) e se } \\
\text { podem ser adiados com } \\
\text { segurança. }\end{array}$ \\
\hline
\end{tabular}

Fonte: Autoria própria, 2021.

RC: 92275

Disponível em: https://www.nucleodoconhecimento.com.br/saude/surto-psicotico 
Dentre os estudos em análise, 18,1\% ( $n=2)$ evidenciaram que o tratamento intensivo constitui conduta imprescindível na abordagem do surto psicótico. Estudo de Chang et al. (2015) demonstra que os pacientes em primeiro episódio de surto psicótico que receberam um ano adicional de intervenção especializada precoce desenvolveram menos sintomas negativos e depressivos e apresentaram uma taxa de abandono menor no tratamento ambulatorial.

Em consonância, pesquisa de Jong et al. (2019) aponta o tratamento pelo programa intensivo de grupo multimodal (FACT Plus) como superior ao manejo usual. Esta proposta de tratamento formatou sessões grupais de acompanhamento de 90 minutos por 9 meses e sessões semanais pelos 3 meses seguintes. Tais encontros objetivaram desenvolver atividades de psicoeducação, planejamento de recuperação, tomada de decisão compartilhada, elaboração de um plano de crise, monitoramento da adesão à terapia medicamentosa, atividades de ressocialização, intervenções familiares e intervenções esportivas e de estilo de vida. Neste programa observou-se que os pacientes com transtornos psicóticos que apresentavam risco de reinternação passaram 19,4 dias a menos (56,1\%) em internação psiquiátrica do que os pacientes em tratamento usual.

Além do tratamento intensivo, observou-se que o tratamento integrado, motivacional e com elementos terapêuticos psicoeducativos e comportamentais e agregado à terapia psicossocial de grupo também desencadeou interações positivas na abordagem dos surtos. Neste sentido, o estudo de Gouzoulis-Mayfrank et al. (2015) investigou o manejo de um grupo de pacientes com sintomas psicóticos e, dentre os quais, atestou-se que aqueles que participaram do programa de abordagem integrada vincularam-se mais satisfatoriamente ao tratamento.

Ademais, $18,1 \%$ dos artigos selecionados $(n=2)$ expuseram a relevância da terapia de recuperação social. Dentre eles, o ensaio clínico randomizado de fase II de Lowen et al. (2020) submeteu 75 pacientes à divisão em um grupo-intervenção e um grupo-controle. Neste contexto, o primeiro grupo foi submetido à STR (Terapia de Recuperação Social) utilizando técnicas de terapia cognitivo-comportamental com

RC: 92275

Disponível em: https://www.nucleodoconhecimento.com.br/saude/surto-psicotico 
administração de casos e suporte de emprego. Na conclusão do acompanhamento atestou-se que as técnicas comportamentais melhoraram os resultados funcionais em pacientes com primeiro episódio de sintomatologia psicótica, objetivo primordial da STR.

No que tange às terapias de remediação cognitiva de pacientes em surto psicótico, desfechos favoráveis foram observados em dois estudos $(18,1 \%$ dos artigos selecionados). Neste cenário, ensaio clínico randomizado de Ventura et al. (2017) analisa a utilização de RC (Remediação Cognitiva) associada a medicações antipsicóticas e acompanhamento psiquiátrico em 80 pacientes, dentre os quais aferiu-se que no grupo de intervenção houve desenvolvimento importante de habilidades cognitivas e melhora do funcionamento social, assim como redução significativa de sintomas negativos e de fatores de risco para cronicidade da psicose.

Estudo norte-americano de Kane et al. (2015) evidencia a intervenção com um programa de tratamento precoce de episódios psicóticos (RAISE-ETP), o qual combina fármacos e tratamentos psicossociais fornecidos por profissional devidamente treinado e equipe multidisciplinar, com o fito de melhorar substancialmente o resultado funcional e a qualidade de vida de pacientes com primeiro episódio de psicose. Este tratamento pode ser realizado em ambientes clínicos e demonstrou poder alterar a trajetória da esquizofrenia em sua fase inicial. Em conformidade, pesquisa de Wessels et al. (2017) também aborda a resposta a tratamentos psicológicos (terapia cognitivo-comportamental e aconselhamento de suporte) precocemente instituídos em pessoas predisponentes a primeiros surtos de psicose. Neste estudo, 128 pessoas foram submetidas à realização de terapia cognitivo-comportamental (TCC) e aconselhamento de suporte e, dentre as quais, as mais jovens beneficiaram-se com a realização do tratamento precoce.

Constatou-se, a partir da análise dos estudos selecionados, que a inserção de curandeiros tradicionais e religiosos dentro do sistema de saúde, como adjuvantes aos cuidados destes pacientes psiquiátricos, também demonstrou ser uma prática positiva. Segundo Gureje et al. (2017), há a necessidade de aferir um método de

RC: 92275

Disponível em: https://www.nucleodoconhecimento.com.br/saude/surto-psicotico 
incorporação dos serviços dos curandeiros ao sistema de saúde pública convencional. No entanto, para isto, são necessárias evidências empíricas da viabilidade e da eficácia de um envolvimento formal dos curandeiros com o sistema público de saúde. Tais evidências iriam, dentre outros contextos, avaliar de que maneira este cuidado prestado poderia atuar no que diz respeito à redução do uso de práticas de tratamento nocivas e abusivas.

Por fim, ensaio randomizado e controlado por placebo de O'Donoghue et al. (2018) avaliou uma proposta de tratamento psicossocial para a qual 90 jovens de 15 a 24 anos de idade com transtornos psicóticos foram recrutados. Verificou-se, neste trabalho, a necessidade de avaliar se medicamentos antipsicóticos seriam indicados para todos os jovens com primeiro episódio de psicose e se o tratamento farmacológico poderia ser adiado de forma segura.

\section{DISCUSSÃO}

\section{MANEJO DO PACIENTE EM PRIMEIRO EPISÓDIO DE SURTO PSICÓTICO}

A partir da leitura e análise dos resultados, observa-se que diversas condutas foram sugeridas como positivas para o paciente em primeiro episódio de surto psicótico. Entre as terapias mais relevantes estavam o tratamento intensivo, a terapia integrada, a terapia de remediação cognitiva e a terapia de recuperação social.

Nesse sentido, o estudo de Chang et al. (2015) que discute a duração ideal da intervenção precoce para o PEP (primeiro episódio psicótico), apontou que o tratamento intensivo, isto é, por mais de 24 meses, de forma especializada foi consideravelmente melhor em relação aos pacientes do grupo controle, que por sua vez receberam o tratamento padrão.

Ademais, o estudo proposto por Jong et al. (2019) também concluiu que programa intensivo, como FACT Plus, que envolvia psicoeducação, ressocialização, atividades

RC: 92275

Disponível em: https://www.nucleodoconhecimento.com.br/saude/surto-psicotico 
esportivas, planejamento, adesão ao tratamento farmacológico e entre outras, melhorou o tempo de internação de pacientes psiquiátricos em relação àqueles que só utilizavam a farmacoterapia e acompanhamento com especialista.

Desse modo, tais descobertas convergem aos estudos de Bertelsen et al. (2008), que comparou pacientes em PEP no OPUS (programa intensivo de intervenção precoce) e em tratamento tardio, que consistia em centros comunitários de saúde mental. $O$ autor concluiu que os pacientes submetidos ao programa intensivo mostraram sinais mais significativos após 2 anos, a exemplo de melhora dos sintomas psicóticos, adesão ao tratamento, doses menores de fármacos psicóticos, maior satisfação, entre outros. Assim, verifica-se que terapias intensivas podem ser uma das condutas a serem tomadas em pacientes no primeiro episódio de surto psicótico.

Em relação à terapia integrada, os achados de Gouzoulis e seus colaboradores (2015), foram positivos ao utilizarem tratamento normal com terapias específicas em grupo, unindo elementos psicoeducativos e comportamentais nos pacientes com psicose e vício. Dessa forma, observou-se que o grupo intervenção apresentou maior satisfação e mudança no uso de substâncias em comparação ao grupo controle, que recebeu a intervenção hospitalar padrão. Nesse sentido, o Lecardeur (2013) com o resultado de seu estudo. verificou os benefícios da terapia integrada, no caso em questão foi a terapia cognitiva comportamental em combinação com fármacos apropriados. Pessoas que passaram por um PEP reduziram a sintomatologia psicológica e apresentaram melhora na qualidade de vida em comparação ao tratamento usual de forma isolada. Desse modo, pode-se verificar que práticas integrativas podem ser benéficas para esses pacientes.

Os resultados também apresentaram relevância acerca da terapia de remediação cognitiva (CRT). Ventura et al. (2017), analisou o uso de medicações com RC (remediação cognitiva) e observou que o grupo intervenção apresentou redução de sintomas negativos e de agentes que podem levar à cronicidade da psicose.

RC: 92275

Disponível em: https://www.nucleodoconhecimento.com.br/saude/surto-psicotico 
Também apresentaram melhora das habilidades cognitivas e do funcionamento social.

Já Datta e seus contribuintes (2020), observou que a remediação cognitiva contribuiu positivamente no funcionamento cognitivo de pacientes, em comparação ao tratamento usual, mas que em relação ao estado mental geral o tratamento usual demonstrou-se ser mais favorável.

Já em relação a CRT em adolescentes com esquizofrenia de início precoce, Puig et al. (2014) em seu estudo mostrou melhora significativa em comparação ao tratamento usual, pois os adolescentes apresentaram melhoras cognitivas, como a memória verbal e memória de trabalho. Assim, verifica-se que a terapia de remediação cognitiva pode ser útil no tratamento desses pacientes.

A terapia de recuperação social foi abordada no estudo de Lowen e colaboradores (2020), no qual foi averiguado que os pacientes que foram submetidos à SRT (terapia de recuperação social) demonstraram melhores resultados no primeiro episódio psicótico. Essa prática abrange técnicas da TCC (terapia cognitivocomportamental) somado a suporte de emprego e coordenação de casos, isto é, uma adaptação da TCC propriamente dita. O estudo de Fowler et al (2017) vai de encontro ao resultado obtido, o autor expôs que a SRT é útil para jovens com primeiro PEP, pois propicia melhores resultados funcionais, haja vista que o indivíduo se envolverá socialmente. Dessa maneira, verifica-se que a terapia de recuperação social é clinicamente benéfica aos pacientes em primeiro surto psicótico, pois propiciou ao paciente maior aumento do seu tempo gasto em atividades em comparação aos que receberam os serviços usuais, e consequentemente apresentaram melhores resultados.

O tratamento precoce também foi um dos manejos abordados. Os pacientes mais jovens com alto risco clínico $(\mathrm{CRH})$ foram beneficiados com o tratamento de forma precoce nos primeiros surtos psicóticos. O tratamento em questão foi TCC em conjunto com aconselhamento de suporte. Contudo, foi demonstrado que os

RC: 92275

Disponível em: https://www.nucleodoconhecimento.com.br/saude/surto-psicotico 
indivíduos com sintomas negativos e baixo funcionamento poderão necessitar de tratamentos adicionais, ou seja, o tratamento em questão utilizado precocemente é mais notável nos pacientes mais jovens com CRH e bom funcionamento (WESSELS et al., 2017).

De acordo com Kane et al. (2015), através do RAISE (programa de tratamento precoce de primeiros episódios de surto psicótico) verificou-se que o tratamento integrado como defendido por Gouzoulis et al. (2015), com auxílio de equipe multidisciplinar, tratamentos psicossociais, farmacoterapia, podem impactar positivamente a vida do paciente em PEP. Nesse sentido, pode-se verificar que o tratamento precoce não propicia somente benefícios clínicos, mas também econômico, haja vista que reduzem sintomas e progressão patológica a partir do primeiro episódio de surto psicótico (NICHOLL et al., 2010).

Ademais, o estudo de O'Donoghue et al. (2018) traz à vista se é ou não benéfico o uso de fármacos antipsicóticos em jovens do primeiro episódio de surto psicótico. Nesse sentido, o autor conclui que se faz necessário diretrizes que determinem de forma mais esclarecedora acerca da utilidade de fármacos antipsicóticos em jovens no PEP e informe o tratamento conforme o estágio do paciente. Schmidt et al. (2019), por sua vez, expõe a importância de protocolos que permitam identificar intervenções mais eficazes a respeito da transição do estado clínico de psicose. Essa questão foi abordada por Francey et al. (2010), seu estudo questionou quem necessitava de medicamentos antipsicóticos nos estágios iniciais de psicose, ele conclui então que depende dos sintomas de cada indivíduo e que inicialmente é válido utilizar a terapia psicossocial, haja vista que medicamentos antipsicóticos podem ser eficazes em casos agudos, porém apresentam efeitos colaterais. Assim, segundo o autor, esses fármacos só devem ser introduzidos quando a primeira etapa falhar ou quando o paciente se tornar mais agressivo.

RC: 92275

Disponível em: https://www.nucleodoconhecimento.com.br/saude/surto-psicotico 


\section{PROGNÓSTICO DO PACIENTE EM PRIMEIRO EPISÓDIO DE SURTO PSICÓTICO}

Inúmeras intervenções e terapêuticas foram estabelecidas e citadas como benéficas no manejo do paciente em primeiro episódio de surto psicótico. De acordo com o estudo de Chang e colaboradores (2015), os pacientes que receberam um ano adicional de intervenção especializada precoce após o episódio de surto psicótico, ou seja, o grupo intervenção, apresentaram funcionamento significativamente melhor, menos sintomas negativos e depressivos e uma taxa de abandono menor no tratamento ambulatorial do que o grupo de controle. Com relação ao resultado funcional, algumas ressalvas foram feitas. Os pacientes de ambos os grupos, controle e intervenção, exibiram um grau moderado de incapacidade funcional no início do estudo. Os pacientes do grupo de intervenção apresentaram melhora funcional progressiva, ao passo que os do grupo controle não apresentaram melhora significativa da função ao longo de 12 meses.

O status de recuperação funcional alcançado no curso inicial da doença (precoce) demonstra que, em longo prazo, será possível observar resultados no funcionamento social e na diminuição de sintomas negativos (ÁLVAREZ-JIMÉNEZ et al., 2012). Ademais, a melhora dos sintomas negativos e depressivos é de importância clinica crítica, uma vez que sintomas depressivos ocorrem frequentemente em pessoas com transtornos psicóticos e estão intimamente associados a um risco elevado de suicídio (COTTON et al., 2012).

Se tratando de tratamento hospitalar da psicose, a utilização de tratamento integrado, motivacional e com elementos terapêuticos psicoeducativos e comportamentais foi muito útil (GOUZOULIS-MAYFRANK et al., 2015). O estudo de Gouzoulis-Mayfrank e colaboradores (2015) mostrou que pacientes do grupo de intervenção, que receberam terapêutica integrada, desenvolveram maior motivação para a abstinência do que os do grupo controle e reduziram transitoriamente o uso

RC: 92275

Disponível em: https://www.nucleodoconhecimento.com.br/saude/surto-psicotico 
de substâncias em maior extensão. Dessa forma, é possível afirmar que a terapia integrada é de grande auxílio em um possível abandono do uso de substâncias.

Em pacientes com diagnóstico duplo (psicose e uso de substâncias), a terapia integrada mostra sucessos pequenos e parciais (CLEARY et al., 2008). Além disso, programas de tratamento com mais de um ano de duração tem se mostrado mais eficazes, em comparação com períodos de tratamento mais curtos, pois os pacientes com diagnóstico duplo se estabilizam e se recuperam apenas gradualmente, ao longo de um período de vários anos (DRAKE; O'NEAL, WALLACH, 2008). Considerando que o estudo de Gouzoulis-Mayfrank e colaboradores (2015) utilizou um período de intervenção de 5 meses, existe a possibilidade dos resultados se perderem a longo prazo.

A terapia cognitiva também tem se mostrado muito eficaz na reabilitação de pacientes em primeiro surto psicótico. De acordo com Datta e colaboradores (2020), o uso da terapia de remediação cognitiva pode ajudar a melhorar a memória de curto prazo (uma função cognitiva). No entanto, para obter uma melhora do estado mental, a terapia usual apresenta mais resultados positivos. A terapia de grupo também apresentou resultados positivos na melhora do estado global (melhora da memória em curto prazo e do estado mental).

A terapia cognitiva foi hipotetizada como uma intervenção potencialmente válida para indivíduos em primeiro surto psicótico, pois: é mais aceita, tolerada e promove menos estigma quando comparada a outras intervenções, por exemplo, a psicofarmacologia; não expõe o grupo de falsos positivos (que não iria evoluir para psicose) aos efeitos adversos das medicações antipsicóticas; é efetiva no tratamento de sintomas inespecíficos, como depressão e ansiedade (KIM et al., 2011).

O uso de remediação cognitiva também tem se mostrado eficaz na reabilitação psiquiátrica de um paciente em primeiro episódio de surto psicótico. O estudo de Ventura e colaboradores (2017) demonstra que pacientes que passaram pela remediação cognitiva mostraram melhorias significativas nos sintomas negativos,

RC: 92275

Disponível em: https://www.nucleodoconhecimento.com.br/saude/surto-psicotico 
que incluem déficit na fala e na afetividade, expressados por meio do isolamento, falta de iniciativa, dificuldade de demonstrar sentimentos e diminuição da fala, ao longo de um período de 12 meses. Uma metanálise feita no mesmo ano indicou que a remediação cognitiva teve efeitos benéficos sobre os sintomas negativos, mas o tamanho do efeito foi pequeno a moderado (Cella et al., 2017). Além disso, uma meta-análise em pacientes com primeiro episódio de psicose mostrou que a remediação cognitiva produz melhorias pequenas, mas estatisticamente significativas em alvos secundários, como sintomas e funcionamento (REVELL et al., 2015).

Em um relato de experiência, o estudo de Santos, Rodriguez e Fay (2014), constatou que a abordagem grupal constitui uma modalidade rica de sentidos possíveis de proporcionar condições em termos de prevenção e promoção de saúde aos pacientes em primeiro surto psicótico. Os fenômenos específicos do grupo demonstraram ser agregadores e, portanto, consideráveis para o cuidado com o sofrimento psíquico. Ademais, no estudo de Lowen e colaboradores (2020), que utilizou o SUPEREDEN3 (ensaio clínico randomizado de fase II), sugere-se que a terapia de recuperação social (SRT), ou seja, em grupo, é útil para melhorar os resultados funcionais em pessoas com primeiro episódio psicótico.

Uma intervenção multimodal, ou seja, utilizando diversos parâmetros de tratamento, também se mostra eficaz no tratamento de pacientes em primeiro surto psicótico. $O$ uso do Programa intensivo de grupo multimodal FACT Plus mostrou resultados promissores na diminuição do tempo de permanência em hospitalização psiquiátrica (LOS), ou seja, o uso do programa auxilia diretamente em uma recuperação mais rápida de um episódio de psicose (JONG et al., 2019). De acordo com Pitschel-Walz e colaboradores (2006), que também utilizaram uma abordagem multimodal, os dias de internação do paciente reduziram significativamente e também foi observado uma maior adesão a medicamentos.

Além disso, o uso de uma equipe multimodal, ou seja, multidisciplinar, também é benéfico no tratamento do primeiro episódio de surto psicótico. O programa de

RC: 92275

Disponível em: https://www.nucleodoconhecimento.com.br/saude/surto-psicotico 
tratamento precoce de primeiro episódio de surto psicótico (RAISE) pode ser realizado em ambientes clínicos e impactar diretamente no processo clínico desses casos (KANE et al., 2015). Ademais, de acordo com Lowen e colaboradores (2019), o uso de uma Prevenção multimodal do primeiro episódio psicótico por meio de N-acetil-I-cisteína e/ou o uso de uma intervenção psicológica preventiva integrada podem ser eficazes e toleráveis com relação à transição para psicose, bem como podem oferecer melhorias no funcionamento social.

Em muitos países de renda baixa e média (LMIC), os curandeiros tradicionais e religiosos (provedores de saúde alternativos complementares - CAPs) são os principais prestadores de cuidados às pessoas afetadas com surto psicótico. $O$ estudo de Gureje e colaboradores (2017) aponta que o uso dos CAPs no tratamento de desordens mentais pode ser benéfico ou não, sendo que suas modalidades de tratamento podem ser amplamente agrupadas em (1) prejudicial, (2) inócuo e (3) possivelmente útil. As práticas prejudiciais incluem algemas, espancamentos / agressões físicas, escarificação, longos períodos de jejum (fome) impostos e isolamento.

Assim, o estudo sugere o compartilhamento de tarefas entre CAPs e prestadores de cuidados de saúde primários (PHCPs), tais como médicos, enfermeiros, dentre outros. Esta abordagem de compartilhamento de tarefas foi implantada em vários graus de eficácia na tentativa de expandir os serviços para diferentes condições, tais como as desordens mentais, como surtos psicóticos (ORGANIZAÇÃO MUNDIAL DE SAÚDE, 2008).

A análise de preditores de resposta ao tratamento por meio de intervenções psicológicas, tais como variáveis demográficas, sintomáticas e funcionais que predizem melhora nos sintomas positivos, negativos e básicos em pessoas com alto risco clínico de primeiro episódio de psicose também deve ser feito. Constatou-se que pessoas que viviam de forma independente, eram mais jovens e apresentavam funcionamento basal mais alto, mostraram mais melhora nos resultados sintomáticos no acompanhamento psicológico (WESSELS et al., 2017).

RC: 92275

Disponível em: https://www.nucleodoconhecimento.com.br/saude/surto-psicotico 
A formulação das estratégias de intervenção precoce, segundo as quais o cuidado clínico nos momentos iniciais seria efetivo, proporciona prognósticos mais positivos de recuperação da doença. A intenção dos primeiros serviços é oferecer assistência psiquiátrica aos casos iniciais e reduzir o prejuízo causado pela iatrogenia resultante do longo tempo de espera e sofrimento antes da primeira tentativa de buscar ajuda especializada (FREITAS-SILVA LR; ORTEGA, 2014).

Por fim, o estudo de O'Donoghue e colaboradores (2018) avalia ainda se os medicamentos antipsicóticos são indicados para todos os jovens com primeiro episódio de surto psicótico, e se a recuperação na psicose é possível sem o uso de medicação antipsicótica, ou seja, se um grupo de primeiro episódio de psicose (FEP) recebendo apenas intervenções psicossociais intensivas pode alcançar remissão sintomática e recuperação funcional. Além disso, a contribuição relativa da doença psicótica e da medicação antipsicótica em termos de alterações estruturais do cérebro também deve ser elucidada.

\section{CONCLUSÃO}

Conclui-se a importância e a necessidade de compreender os fatores causais do surto psicótico, bem como a especialização de conduzir os primeiros episódios do surto para efeitos de um desfecho desfavorável do paciente.

O estudo evidenciou a relevância de um atendimento multidisciplinar, atuante no acompanhamento terapêutico associado, em alguns casos, a fármacos. Além disso, ressalta-se a necessidade de atentar diagnósticos adicionais para condução do caso, assim como o prognóstico do paciente em questão.

Salienta-se que é importante permanecer a realização de estudos para readequar manejos mais eficientes na condução do paciente e as diversas formas de atuação na prevenção e no mapeamento de possíveis casos de surto psicótico.

RC: 92275

Disponível em: https://www.nucleodoconhecimento.com.br/saude/surto-psicotico 


\section{REFERÊNCIAS}

ÁLVAREZ-JIMÉNEZ, M., GLEESON, J. F., HENRY, L. P., et al. Road to full recovery: longitudinal relationship between symptomatic remission and psychosocial recovery in first-episode psychosis over 7.5 years. Psychol Med. 2012;42(3):595606.

BERTELSEN, M. et al. Five-Year Follow-up of a Randomized Multicenter Trial of Intensive Early Intervention vs Standard Treatment for Patients With a First Episode of Psychotic Illness. Arch gen psychiatry. v. 65 n. 2008

CHANG, W. C., et al. Optimal duration of an early intervention programme for firstepisode psychosis: Randomised controlled trial. The British Journal of Psychiatry, v. 206, ed. 6, p. 492-500, 2 jan. 2018. DOI: 10.1192/bjp.bp.114.150144. Disponível em: https://www.cambridge.org/core/journals/the-british-journal-ofpsychiatry/article/optimal-duration-of-an-early-intervention-programme-forfirstepisode-psychosis-randomised-controlledtrial/25D326D813DDCC6CA129BF80B25E41B0\#article. Acesso em: 12 maio 2021.

CLEARY, M. et al. Psychosocial interventions for people with both severe mental illness and substance misuse. Cochrane Database Syst Rev. 2008; Disponível em: https://pubmed.ncbi.nlm.nih.gov/24092525/; Acesso em: 1 junho 2021.

COTTON, S. M. et al. Depressive symptoms in first episode schizophrenia spectrum disorder. Schizophr Res. 2012;134(1):20-26.

DATTA, S. S.; DARUVALLA, R.; KUMAR, A. Psychological interventions for psychosis in adolescents. Cochrane Database of Systematic Reviews, n. CD009533, ed. 7, 3 jul. 2020. DOI 10.1002/14651858.CD009533.pub2. Disponível em:

https://www.cochranelibrary.com/cdsr/doi/10.1002/14651858.CD009533.pub2/full. Acesso em: 12 maio 2021.

RC: 92275

Disponível em: https://www.nucleodoconhecimento.com.br/saude/surto-psicotico 
DE JONG, M. H. et al. An intensive multimodal group programme for patients with psychotic disorders at risk of rehospitalization: a controlled intervention study. BMC Psychiatry, v. 19, ed. 241, p. 1-6, 5 ago. 2019. DOI https://doi.org/10.1186/s12888019-2229-x. Disponível em: https://bmcpsychiatry.biomedcentral.com/articles/10.1186/s12888-019-2229x\#citeas. Acesso em: 12 maio 2021.

DRAKE, R. E.; O'NEAL, E. L.; WALLACH, M. A. A systematic review of psychosocial research on psychosocial interventions for people with co-occurring severe mental and substance use disorders. J Subst Abuse Treat. 2008;34(1):123-138. Disponível em: https://pubmed.ncbi.nlm.nih.gov/17574803/; Acesso em: 1 junho 2021.

FOWLER, D. et al. Social recovery therapy in combination with early intervention services for enhancement of social recovery in patients with first-episode psychosis (SUPEREDEN3): a single-blind, randomised controlled trial. Lancet Psychiatry. December 2017.

FRANCEY, S. M. et al. Who needs antipsychotic medication in the earliest stages of psychosis? A reconsideration of benefits, risks, neurobiology and ethics in the era of early intervention. Schizophrenia Research. March 2010.

FREITAS-SILVA, L. R.; ORTEGA, F. Intervenção precoce na psicose: de estratégia clínica a possível categoria diagnóstica. Psicol Estud. 2014; 19:729-39. Disponível em: $\quad$ https://www.scielo.br/j/pe/a/Cyv6z5P5tDZd3Bvp9Vhjt3p/abstract/?lang=pt; Acesso em: 3 junho 2021.

GOUZOULIS-MAYFRANK, E. et al. Trans-Sector Integrated Treatment in Psychosis and Addiction: A randomized controlled study of a motivational, cognitive behavioral therapy program under standard hospital treatment conditions. Deutsches Ärzteblatt Online, Alemanha, v. 112, n. 41, p. 683-691, 9 out. 2015. DOI 10.3238/arztebl.2015.0683. https://www.aerzteblatt.de/int/archive/article/172416. Acesso em: 12 maio 2021.

RC: 92275

Disponível em: https://www.nucleodoconhecimento.com.br/saude/surto-psicotico 
GUREJE, O. et al. COllaborative Shared care to IMprove Psychosis Outcome (COSIMPO): study protocol for a randomized controlled trial. BMC - Trials, v. 18, n. 462, p. $1-15,10$ out. 2017. DOI https://doi.org/10.1186/s13063-017-2187-x. Disponível em: https://trialsjournal.biomedcentral.com/articles/10.1186/s13063-0172187-x. Acesso em: 12 maio 2021.

KANE, J. M. et al. The RAISE early treatment program for first-episode psychosis: background, rationale, and study design. The Journal of Clinical Psychiatry, v. 76, n. 3, p. 240-246, 2015. DOI 10.4088 / JCP.14m09289. Disponível em: https://www.researchgate.net/publication/274401145_The_RAISE_Early_Treatment_ Program_for_First-Episode_Psychosis. Acesso em: 12 maio 2021.

KIM, K. R. et al. Clinical efficacy of individual cognitive therapy in reducing psychiatric symptoms in people at ultra-high risk for psychosis. Early Intervention Psychiatry, 2011. 5(2),174-178. Disponível em: https://pubmed.ncbi.nlm.nih.gov/21535425/; Acesso em: 2 junho 2021.

LECARDEUR, L. Thérapies cognitives et comportementales après les premiers épisodes psychotiques. L’Encéphale. v. 39 n. 2, Septembre 2013.

LOWEN, C. et al. Measuring adherence in social recovery therapy with people with first episode psychosis.Behav Cogn Psychother, v. 48, n. 1, p. 82-90, jan. 2020. DOI 10.1017/S1352465819000432. Disponível em: https://pubmed.ncbi.nlm.nih.gov/31379311/. Acesso em: 12 maio 2021.

NICHOLL, D. et al. Burden of schizophrenia in recently diagnosed patients: Healthcare utilisation and cost perspective. Curr Med Res Opin. April 2010.

O'DONOGHUE, B. et al. Staged treatment and acceptability guidelines in early psychosis study (STAGES): A randomized placebo controlled trial of intensive psychosocial treatment plus or minus antipsychotic medication for first-episode psychosis with low-risk of self-harm or aggression. Study protocol and baseline

RC: 92275

Disponível em: https://www.nucleodoconhecimento.com.br/saude/surto-psicotico 
characteristics of participants. Early Intervention in Psychiatry, v. 13, n. 4, p. 953960, ago. 2019. DOI 10.1111/eip.12716. Disponível em:https://pesquisa.bvsalud.org/portal/resource/pt/mdl-30024100. Acesso em: 12 maio 2021.

PITSCHEL-WALZ, G. et al. Psychoeducation and compliance in the treatment of schizophrenia: results of the Munich psychosis information project study. J Clin Psychiatry. 2006;67:443-52.

Disponível em: https://pubmed.ncbi.nlm.nih.gov/16649832/; Acesso em: 2 junho 2021.

PUIG, O. et al. Cognitive Remediation Therapy in Adolescents With Early-Onset Schizophrenia: A Randomized Controlled Trial. Journal of the american academy of child \& adolescent psychiatry. V. 53 n. 8, August 2014.SANTOS, Janice Dombrovski dos; RODRIGUEZ, S. Y. S.; FAY, Eliane Moreira. Grupoterapia com pacientes psicóticos: relato de uma experiência. Rev. SBPH, Rio de Janeiro , v. 17, n. 2, p. 137-148, dez. 2014 . Disponível em $<$ http://pepsic.bvsalud.org/scielo.php?script=sci_arttext\&pid=S151608582014000200009\&lng=pt\&nrm=iso>. Acesso: em 01 jun. 2021.

SCHMIDT, S. J. et al. Multimodal prevention of first psychotic episode through $\mathrm{N}$-acetyl-I-cysteine and integrated preventive psychological intervention in individuals clinically at high risk for psychosis: Protocol of a randomized, placebo-controlled, parallel-group trial. Early Intervention in Psychiatry, v. 13, n. 6, p. 1404-1415, 13 dez. 2019. DOI 10.1111/eip.12781. Disponível em: https://pubmed.ncbi.nlm.nih.gov/30784233/. Acesso em: 12 maio 2021.

VENTURA, J. et al. Cognitive remediation can improve negative symptoms and social functioning in first-episode schizophrenia: a randomized controlled trial. Schizophrenia Research, v. 203, p. 24-31, 9 nov. 2019. DOI 10.1016/j.schres.2017.10.005. Disponível em: https://www.ncbi.nlm.nih.gov/pmc/articles/PMC6589092/. Acesso em: 12 maio 2021.

RC: 92275

Disponível em: https://www.nucleodoconhecimento.com.br/saude/surto-psicotico 
WESSELS, H. et al. Predictors of treatment response to psychological interventions in people at clinical high risk of first-episode psychosis. Early Intervention in Psychiatry, v. 13, n. 1, p. 120-127, jul. 2017. DOI 10.1111 / eip.12460. Disponível em:

https://www.researchgate.net/publication/318200477_Predictors_of_treatment_respo nse_to_psychological_interventions_in_people_at_clinical_high_risk_of_firstepisode_psychosis. Acesso em: 12 maio 2021.

WORLD HEALTH ORGANIZATION, PEPFAR \& UNAIDS. (2007). Task shifting : rational redistribution of tasks among health workforce teams: global recommendations and guidelines. World Health Organization.

Enviado: Julho, 2021.

Aprovado: Julho, 2021.

RC: 92275

Disponível em: https://www.nucleodoconhecimento.com.br/saude/surto-psicotico 